UDC 631.358.44/45

(C) 2017

V. Adamchuk, Academician of NAAS, Doctor of Engineering National Science Center "Institute of Mechanization and Electrification Agriculture”

V. Bulgakov, Academician of NAAS, Doctor of Engineering

I. Golovach, Doctor of Engineering

S. Smolinskyi, Candidate of Technical Sciences

National University of Bioresources and nature use of Ukraine Ignatiev Ye.

Tavria state agrotechnological university

\title{
THEORY OF STROKE INTERACTION OF A TUBER OF POTATO AT SEPARATION OF
}

The purpose. To raise efficiency and quality of separation of potato heap by theoretical substantiation of rational parameters of separator, proceeding from conditions of non-damaging tubers at their stroke interaction. Methods. Theoretical researches of stroke interaction were spent with application of substantive provisions of higher mathematics, theoretical mechanics, methods of formulation of programs for numerical calculations on the personal computer and construction of graphical dependences, and also analysis of the gained results. Results. Equivalent scheme of stroke interaction of a tuber of potato with elastic surface of reflecting transporter is developed. In view of quotient of recovery of speed of a tuber at impact analytical forms for determination of value, direction and speed of a tuber after impact are gained. It has enabled on the basis of application of principle of momentum at impact to gain analytical forms for determination of stroke impulse and force of impact of a tuber with elastic surface of reflecting transporter. Conclusions. New mathematical model of stroke interaction of a tuber of potato is built at separation of heap. On the basis of the gained theoretical results rational kinematic parameters of quality accomplishment of the specified process under condition of non-damaging tubers of potato are probed.

Key words: potato, tuber, separation of heap, stroke interaction, stroke impulse, equivalent scheme.

Formulation of the problem. As you know, potatoes belong to very energy-intensive branches of agriculture. Therefore, when developing new and improving the existing working bodies of potato harvesting machines, it is necessary to ensure a significant reduction in their energy intensity and a substantial increase in the quality of the products obtained with minimal losses in 
harvesting. One of the important technological operations for potato harvesting is the separation of potato tubers, which ensures the purity of the resulting products. Thus, the cleaning working bodies are the main link in ensuring the quality performance of the potato harvesting machine. To this end, a new design of a potato digger separator, which is protected by the Ukrainian patent, will be developed [1].

But experimental studies have shown that some of the potato tubers and soil impurities, with dimensional-mass characteristics similar to the tubers, fly through the cleaning working bodies to the surface of the field or get into the unloading conveyor. This leads to the loss of yield on the one hand and pollution of the resulting products by soil impurities on the other.

Therefore, in order to exclude the flow of tubers through a separator, it is proposed to install a reflective rubber tape smooth conveyor. At the same time, the bulge, which is separated from the separation surface, hits the elastic surface of the reflecting conveyor and returns again to the separating surface. However, as a result of the shock interaction of the tuber with the surface of the conveyor it is possible to damage, which in general can lead to a decrease in the quality of products. Therefore, ensuring that potato tubers are not damaged during shock interaction is a very topical problem, the solution of which is impossible without conducting theoretical studies, in particular the construction of the necessary mathematical model of shock interaction. This will enable to justify the rational kinematic parameters of the separator operation provided that the potato tubers are not damaged during harvesting.

Analysis of recent publications. Separating working bodies used in modern potato harvesting machines should not only provide reliable and qualitative performance of the technological process of cleaning potato tubers from soil impurities, plant remains and clay soils, but also be capable of self-cleaning in the process of work that will ensure appropriate productivity of labor . However, it is known that most systems of separation working bodies used on serial potato harvesters do not always provide a high level of separation of soil impurities [2]. It 
happens, most often, as a result of intense contamination of the cleaning surfaces of the separating working bodies with wet soil.

A lot of scientists and designers worked on the problem of creating effective and reliable in the work of the potato tuber separators when it was assembled, as well as a variety of purifiers used at stationary potato-cleaner points [3-7]. Spiral separators are considered to be the most promising and technologically suitable for the process of cleaning potato tubers when excavated from soil under different soil-climatic conditions. However, despite the large number of technological processes for cleaning the potato tuber during assembly, there is relatively little research on the optimization of the kinematic and structural parameters of the spiral separators. This is especially true in terms of ensuring the conditions of damage to potato tubers in their interaction with spiral waves, reflection from different surfaces and subsequent shock contacts with other working organs.

Purpose. Improvement of efficiency and quality of separation of potato tuber by theoretical substantiation of rational parameters of a separator under conditions of damage of tubers under their shock interaction..

Methods. Теоретичні дослідження ударної взаємодії проводились 3 застосуванням основних положень вищої математики, теоретичної механіки.

Results. The theoretical substantiation of the kinematic parameters of the potato tuber separator was carried out on the basis of constructing a mathematical model of shock interaction of a potato tuber with an elastic surface of a moving reflecting conveyor. For this, first of all, it is necessary to construct an equivalent scheme of the indicated shock interaction (Fig. 1). 


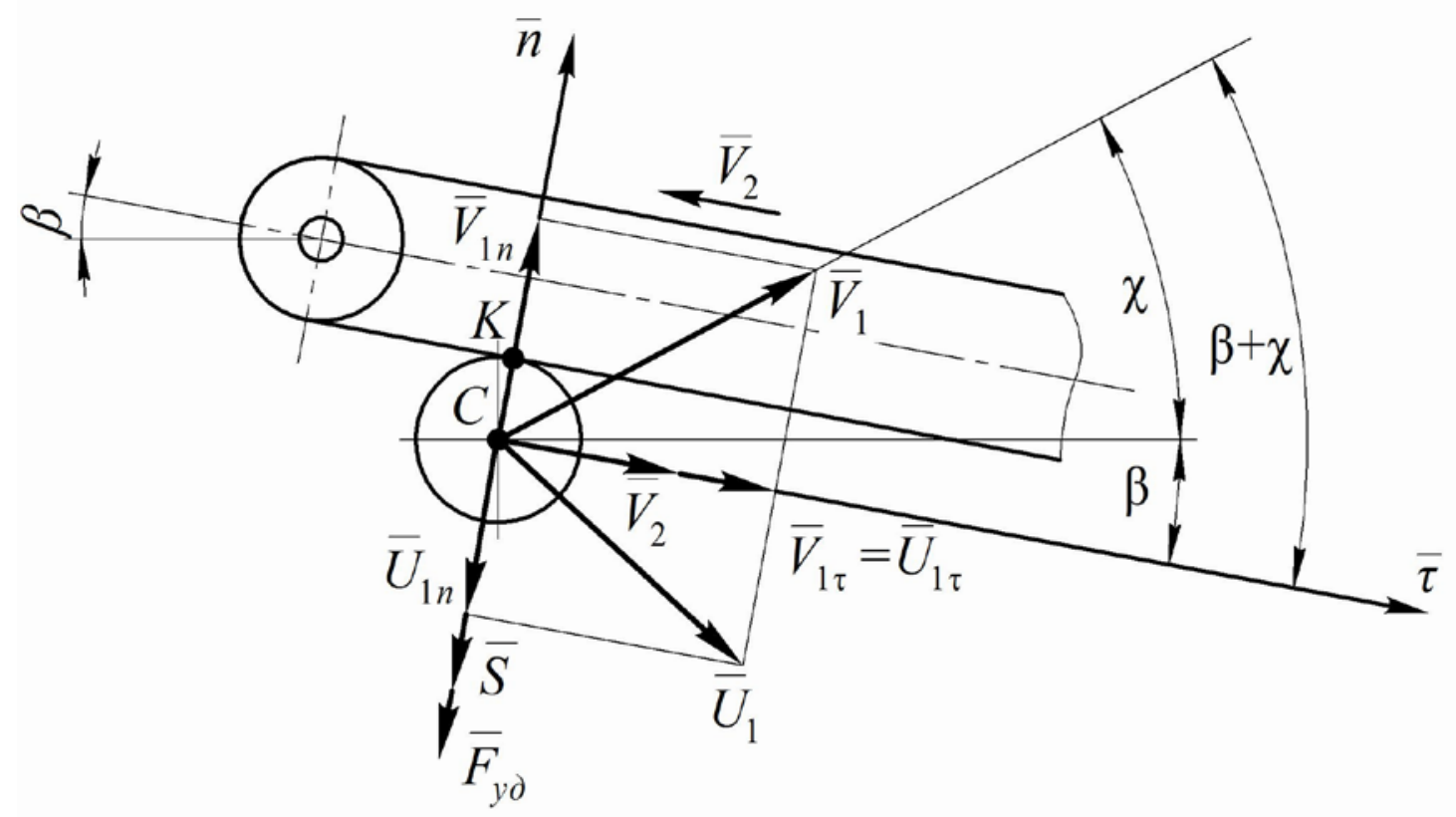

Fig. 1. - Chart of shock interaction potato tubers with a bottom surface of a moving reflecting conveyor

Let's show on an equivalent scheme inclined at an angle $\beta$ to the horizon a moving reflective conveyor, the lower working branch of which is carried out by the shock contact of the potato tuber separating from the separation surface of the separator, and whose trajectory intersects with the lower surface of the conveyor at the point of the impact contact $K$. At the same time, to simplify the analytical calculations, the body of the tubers is approximated ball shape It is obvious that the tuber, in the translational motion on a certain trajectory of the flight in the air, can be carried out under the influence of the moment of frictional force and some other factors acting on the bulb at the moment of its separation from the separating surface, the rotational movement around some of its axes, but with shock interaction the influence of the rotational motion on the magnitude of the shock interaction is not significant. Consequently, in the first approximation, we can assume that the bubble carries only a translational motion along its flight path. In addition, given the small size and mass of the tubers in comparison with the size and weight of the conveyor, it can be considered a material point. Therefore, the forces acting on the bulb at the point $K$ for its contact with the surface of the conveyor during the impact can be considered applied to the center of the mass of 
the tuber (point $C$ ), as shown in Fig.1.

Since the decisive role in the process of this shock contact is played by the normal component of the impact pulse, then for the study of the impact process we choose the natural coordinate system $\bar{\tau} C \bar{n}$ with the origin of the coordinates in the center of the tuber (point $C$ ), axis $\bar{\tau}$ which is directed parallel to the lower branch of the conveyor, and the axis $\bar{n}$ - perpendicular to this branch (in the direction of the normal component of the impact pulse) (Fig. 1).

Let's show on the equivalent scheme the following velocity vectors:

$\overline{V_{1}}$ - bulb speed before impact;

$\bar{V}_{2}$ - speed of conveyor belt;

$\bar{U}_{1}$ - the speed of the tuber after the blow.

In this case, the velocity vector $\bar{V}_{1}$ is directed at an angle $\chi$ to the horizon, and the velocity vector $\bar{V}_{2}$ at an angle $\beta$ to the horizon

As a result, the angle between the vectors $\bar{V}_{1}$ and $\bar{V}_{2}$ is equal to $\beta+\chi$ (Fig. 1).

Let's consider the impact of tubers mass $m$ on a moving conveyor of mass $M$ that moves at a speed $\bar{V}_{2}$. Compared to the mass of the tuber, we can assume that the weight of the conveyor $M \rightarrow \infty$. At the same time, assume that the impact is oblique, partially elastic (coefficient of speed recovery at impact $k<1$ )[8].

Determine the speed of the tuber and conveyer after impact in accordance with the general methodology given in [9], which takes into account the most common case of impact of two bodies having different masses and velocities. According to this method, we determine the projections of the velocities of the tuber and the conveyer to the impact on the coordinate axis $\bar{n}$ and $\bar{\tau}$. According to Fig. We have one:

$$
\begin{aligned}
& V_{1 n}=V_{1} \sin (\beta+\chi), \\
& V_{1 \tau}=V_{1} \cos (\beta+\chi), \\
& V_{2 n}=0, \\
& V_{2 \tau}=V_{2} .
\end{aligned}
$$


Further, according to the same method, we determine the projection of the general velocity $\bar{U}$ both bodies on the axle $\bar{n}$ at the end of an absolutely inelastic blow:

$$
U_{n}=\frac{m V_{1 n}+M V_{2 n}}{m+M} .
$$

Because $V_{2 n}=0$, a $M \rightarrow \infty$, then $U_{n}=0$.

Then projections of tuber and conveyer velocities on the axis $\bar{n}$ and $\bar{\tau}$ after impact will be determined by expressions [9-11]:

$$
\begin{aligned}
& U_{1 \tau}=V_{1 \tau}, \\
& U_{2 \tau}=V_{2 \tau}, \\
& U_{1 n}=U_{n}(k+1)-k V_{1 n}, \\
& U_{2 n}=U_{n}(k+1)-k V_{2 n} .
\end{aligned}
$$

After substituting calculated values of the projections of speeds to impact (1) and values $U_{n}=0$ in (3) we obtain:

$$
\begin{aligned}
& U_{1 \tau}=V_{1} \cos (\beta+\chi), \\
& U_{2 \tau}=V_{2}, \\
& U_{1 n}=-k V_{1} \sin (\beta+\chi), \\
& U_{2 n}=0 .
\end{aligned}
$$

In this case, the velocity modules of the tubers and the conveyor after the impact, respectively, will be equal:

$$
\begin{aligned}
& U_{1}=\sqrt{U_{1 \tau}^{2}+U_{1 n}^{2}}=V_{1} \sqrt{\cos ^{2}(\beta+\chi)+k^{2} \sin ^{2}(\beta+\chi)}, \\
& U_{2}=\sqrt{U_{2 \tau}^{2}+U_{2 n}^{2}}=V_{2} .
\end{aligned}
$$

The directions of the velocities of the bodies after the impact form corners with the normal, tangents of which are equal:

$$
\begin{aligned}
& \operatorname{tg}\left(\bar{n}, \bar{U}_{1}\right)=\frac{U_{1 \tau}}{U_{1 n}}=-\frac{\operatorname{ctg}(\beta+\chi)}{k}, \\
& \operatorname{tg}\left(\bar{n}, \hat{U}_{2}\right)=\frac{U_{2 \tau}}{U_{2 n}}=\infty .
\end{aligned}
$$


Determine further the force of impact of the tuber on the plane of the moving conveyor.

To do this, we apply a theorem on the change in the number of movement of the tuber during the impact, which in the vector form has the form:

$$
m\left(\bar{U}_{1}-\bar{V}_{1}\right)=\bar{S},
$$

where $m$ - mass of tubers; $\overline{\mathrm{s}}$ - shock impulse.

Let us record the equation (7) of the bulb's impact on the moving plane of the conveyor in projections on the axis $\bar{n}$ and $\bar{\tau}$ :

$$
\left.\begin{array}{l}
m\left(U_{1 n}-V_{1 n}\right)=S_{n}, \\
m\left(U_{1 \tau}-V_{1 \tau}\right)=S_{\tau} .
\end{array}\right\}
$$

After substituting (8) above the obtained values of the projections of velocities $U_{1 n}, V_{1 n}, U_{1 \tau}, V_{1 \tau}$ we obtain the following values of projections of the impact pulse on the axis $\bar{n}$ and $\bar{\tau}$ :

$$
\left.\begin{array}{l}
S_{n}=-m V_{1} \sin (\beta+\chi) \cdot(k+1), \\
S_{\tau}=0 .
\end{array}\right\}
$$

Taking into account that the general momentum $S$ is determined from the

$$
\text { expression } \quad S=\sqrt{S_{\tau}^{2}+S_{n}^{2}} \text {, }
$$

we get that $S=S_{n}$, i.e.:

$$
S=-m \cdot(k+1) \cdot V_{1} \sin (\beta+\chi) .
$$

After determining the impact pulse $S$ it is possible to determine in some way the force of impact $F_{y \partial .}$. tubers on a moving conveyor

To do this, we use the integral expression to calculate the impact pulse through the force of impact [8]:

$$
\bar{S}=\int_{t_{0}}^{t_{0}+\Delta t} \bar{F}_{y \partial .} d t
$$

where $\bar{F}_{y d}$. force of impact; $\Delta t-$ stroke duration.

Then, by the theorem on the mean in the integral number, we can write:

$$
S=F_{y \partial . c p .} \Delta t,
$$


where $F_{y d . c p .}$ - average strike force in the time of impact $\Delta t$.

From Expression (13) we find:

$$
F_{y \partial . c p .}=\frac{S}{\Delta t} .
$$

According to [8], the maximum impact force $F_{y d}$ is approximately equal to:

$$
F_{y d}=2 F_{y d . c p .},
$$

or, considering (14),

$$
F_{y \partial .}=\frac{2 S}{\Delta t} .
$$

Substituting expression (11) in expression (16) we obtain:

$$
F_{y d .}=-\frac{2 m(k+1) V_{1} \sin (\beta+\chi)}{\Delta t} .
$$

The sign "-" in expressions (11) and (17) means that the direction of the vectors $\bar{S}$ and $\bar{F}_{y \partial \text {. }}$ is opposite to the direction of the axis $\bar{n}$. But in this case the main thing is the absolute value of these values, so in the following expressions the "-" sign can be omitted.

Obviously, potato tubers are not damaged; the following condition is required:

$$
F_{y \partial .} \leq\left[F_{y \partial .}\right],
$$

where $\left[F_{y d}\right]$ - allowable force of impact of potato tubers on the surface of the conveyor, in which the bulb itself is not damaged.

Consequently, taking into account expression (17), we have the following inequality:

$$
\frac{2 m(k+1) V_{1} \sin (\beta+\chi)}{\Delta t} \leq\left[F_{y \partial}\right] .
$$

From the obtained inequality (19) we find the limit on the value of the speed $V_{1}$ of a potato tuber before the impact on the surface of the conveyor provided it is not damaged: 


$$
V_{1} \leq \frac{\left[F_{y \partial .}\right] \Delta t}{2 m(k+1) \sin (\beta+\chi)} .
$$

Thus, a dynamic condition for the damage to the potato tubers after its impact on the moving surface of the conveyor is obtained..

After calculating the permissible speed of potato tubers before impact, it is possible to determine the structural and kinematic parameters of the separating working organ, which will provide this speed. In [12] an expression was obtained for determining the speed of a potato tuber before impact for a spiral separator:

$$
V_{1}=\sqrt{V_{0}^{2}-2 g H}
$$

where $H$ - the flight height of the bulb from the separating surface to the point of impact contact; $V_{0}$ - the speed of the potato tuber at the moment of separation from the separation surface.

Moreover:

$$
V_{0}=\omega \rho_{i},
$$

where $\omega$ - angular velocity of rotation of the spiral around its longitudinal axis;

$$
\rho_{i}=R_{i}+R_{\sigma},
$$

where $R_{i}$ - radius of a spiral; $R_{\sigma}$ - radius potato tubers.

Taking into account expressions (21), (22) and (23), one can determine the permissible angular speed of the spiral provided that the potato tubers are not damaged:

$$
\omega=\frac{\sqrt{V_{1}^{2}+2 g H}}{R_{i}+R_{\sigma}},
$$

or radius $R_{i}$ spiral at a given angular velocity $\omega$ its rotation:

$$
R_{i}=\frac{\sqrt{V_{1}^{2}+2 g H}}{\omega}-R_{\sigma} .
$$

It is obvious that the theoretical results given in the work can be used in the investigation of the shock interaction of different types of root crops with surfaces of various working bodies of agricultural machines.

\section{Conclusions}


1. A new calculated mathematical model of shock interaction of potato tubers at the separation of a heap.

2. The analytical expressions for calculating the impact pulse and the impact strength of the tuber on the elastic surface of the reflecting conveyor, depending on the kinematic parameters of the separator.

3. Analytical determination of the dynamic limits of the permissible speed of a potato tuber before impact interaction provided that it is not damaged.

4. The theoretical results given in the work can be used in the study of shock interaction of different types of root crops with surfaces of various working bodies of agricultural machines.

\section{Bibliography}

1. Patent of Ukraine № 43907, A01D33 / 08. Wiper blade root bottle fertilizer from impurities / B.M. Bulgakov, P.Yu. Zikov, S.V. Smolinsky, M.G. Berezovy, A.L. Bondarenko. Pubwished Jan. 15, 2002, bullet №1.

2. Petrov G.D. Potato harvesters. - M.: Mashinostroyeniye, 1984. - 320 p.

3. Smolinsky S.V. Experimental determination of parameters of a spiral separator of potato harvesting machines // Agricultural machines. Zb sciences Art., Vip. 8. - Lutsk: Red. dept. LDTU, 2001. ᄀ p. 265-271.

4. Smolinsky S.V. On theoretical calculation of parameters and modes of operation of a spiral separator of potato tuber. // Collection of scientific works of NAU "Mechanization of agricultural production". TomVII K .: In NAU, 2002, p. 290-293.

5. Zaltzman, A., Schmilovitch, Z. Evolution of the potato fluidized bed medium separator // (Conference Paper) American Society of Agricultural Engineers, 1985, 27 p.

6. Ichiki, H. , Nguyen Van, N., Yoshinaga, K. Stone-clod separation and its application to potato cultivation in Hokkaido / Bio-oriented Technology Research Advancement Institution, Engineering in Agriculture, Environment and Food , Volume 6, Issue 2, 2013, P. 77-85. 
7. Karwowski T. Teoria i konstrukcja maszyn rolniczych. T. 3. Warszawa: PWRiL, 1982. - 429 p.

8. Butenin N.V., Luntz Y.L., Merkin D.R. Course of theoretical mechanics. E. 2. - M.: Nauka, 1985. - 496 p.

9. Bat M.I., Dzhanelidze G.Yu., Kelzon A.S. Theoretical mechanics in examples and problems. Vol. 2. - M.: Nauka, 1964. - 664 p.

10. Gieck K., Gieck R. Technische Formelsammlung. - Germering: Gieck Verlag, 1995. - $330 \mathrm{p}$.

11. Byshov N.V., Buryakov Yu.V., Uspensky I.A. Perfection of the technological process of potato harvesters // Sb. employees and post-graduate students, v.2. / RSAA Ryazan, 1996. - P.157-159.

12. Bulgakov V.M., Adamchuk V.V., Golovach I.V., Smolinsky S.V, Ihnatiev Ye.I. The theory of the reflection of potato tubers with the work of a spiral separator. - News of agrarian sciences. - 2017, №11. - P. 55-60. 